\title{
Periodic Data, Burden or Convenience
}

\author{
Bela Stantic \\ Institute for Integrated and Intelligent Systems \\ Griffith University \\ B.Stantic@griffith.edu.au
}

\begin{abstract}
Periodic events seem to be an intrinsic part of our life, and a way of perceiving reality. There are many application domains where periodic data play a major role. In many of such domains, the huge number of repetitions make the goal of explicitly storing and accessing such data very challenging to the extent of even not being possible, in cases of open ended intervals. In this work, we present a concept to represent periodic data in an implicit way. The representation model we propose captures the notion of periodic granularity provided by the temporal database glossary. We define the algebraic operators, and introduce access algorithms to cope with them and also with temporal range queries, proving that they are correct and complete with respect to the traditional explicit approach. In an experimental evaluation we show the advantages of our approach with respect to traditional explicit approach, in terms of space usage, physical disk I/O's and query response time.
\end{abstract}

\section{Introduction}

Periodic data play a major role in many application domains, such as manufacturing, office automation, and scheduling. Day and nights repeat at regular periodic patterns, as well as seasons, and years. Accordingly, many human activities are scheduled at periodic time (e.g., office activities, scheduling of train, airplanes, lessons, etc). Due also to such a wide range of different contexts of application, it is widely agreed that adopting a 'standard' and fixed menu of granularities (e.g., minutes, hours, days, weeks, years and so on in the Gregorian calendric system) is not enough in order to provide the required expressiveness and flexibility. For instance, a while ago Soo and Snodgrass [1] emphasized that the use of a calendar depends on the cultural, legal and even business orientation of the users, and listed many examples of different calendric systems and user-defined periodic granularities, such as academic, legal, and financial year. They also stressed that different user-defined periodic granularities are usually used even in the same area, for example the definition of holidays in different companies. The number of repetitions of periodic data may be very large and in some cases repetitions may also be 'open-ended', meaning that we do not know the ending time when the repetition will stop, which is for example the case in therapies for chronical patients as it may be repeated all the life long. Therefore, in the Computer Science literature (in particular, in the areas of Databases, Logics, and Artificial Intelligence), there is a common agreement that formalisms are needed in order to cope with user-defined periodic data in an implicit (also termed intensional) way, without an explicit storing of all the repetitions. In this work we consider periodic data which have value-equivalent repetitions 
at periodic time (e.g., the periodic schedule of trains); data that are acquired at periodic time, but may assume different values (e.g., periodic monitoring of blood pressure) are not taken into account in this work. Periodic data play an important role in Databases and a specific entry has been devoted to such a topic in the Encyclopedia of Database Systems by Springer. In the Encyclopedia [2], three main classes of Database implicit approaches to user-defined periodicities have been identified:

- Deductive rule-based approaches, using deductive rules, and approaches in classical temporal logics [3].

- Constraint-based approaches, using mathematical formulae and constraints (e.g., [4]), and

- Algebraic also termed Symbolic) approaches, providing a set of 'high-level' and 'user-friendly' operators (e.g., [5], [6], [7], [8], [9], [10]).

A comparison among such classes approaches is out of the scope of this work (the interested reader is referred to [2] and also to [11]). However, it worth stressing that in most approaches in the literature (in particular in all algebraic approaches) the focus is on the design of high-level formalisms to model (in an implicit way) user-defined periodicities in a 'commonsense' or at least 'user-friendly' way. Most of such approaches do not take into account issues such as the definition of relational temporal algebraic operators, extending Codd's operators to query periodic data, range queries and efficient indexing and access of temporal data.

In summary, although there seems to be a general agreement within the Database (and also Artificial Intelligence) literature that general-purpose implicit approaches are needed in order to cope with user-defined periodic data, and despite the fact that a lot of such approaches have been devised in the last two decades, none of such approaches focus specifically on the definition of a comprehensive relational approach coping with user-defined periodic data efficiently, considering a relational representation of periodic data, efficient access of such data, algebraic operators, and additional temporal operators such as range queries. However, all such issues are fundamental for the practical applicability of any Database approach considering periodic data. The goal of our work is to devise such a comprehensive approach. Specifically, our approach has been designed in such a way that:

- The data model has the expressiveness to capture all periodic granularities, as defined in the Database literature [12], [7],

- Algebraic and temporal query operators are correct and complete with respect to the conventional explicit approaches, in which all the repetitions of periodic data are explicitly stored.

- Extended algebraic operators operate in polynomial time, and are a consistent extension of standard nontemporal relational algebraic ones.

First property grants that the expressiveness of our data model is the one requested by the temporal Database literature. Second property grants that, although periodic data are only implicitly stored, we get the same (correct) results as one obtained with traditional (fully explicit) models. Moreover, in this paper we also provide testing, to show the advantages of our approach with respect to conventional explicit approaches, especially in terms of disk I/O's and query response time. 
On the other hand, in this work:

- We do not address the treatment of the transaction time of events (i.e., the time when events are inserted in/deleted from the database [13]) since no periodicity issue is usually involved by it. As a matter of fact, transaction time is orthogonal to valid time (the time when the fact described by the tuples takes place). As a consequence, the proposed approach dealing with the periodic valid time can be extended to deal also with transaction time.

- Although we cope with user-defined periodic granularities we assume that each periodic granularity is directly expressed in terms of a 'bottom' granularity. Therefore, we are not interested to cope with issues concerning conversions between periodic granularities, or properties of relations between them, which is, on the other hand, a main focus of other approaches dealing with multiple granularities, [14], [8], [15], [16].

The rest of the paper is organized as follows. The next Section is a preliminary one, in which we briefly mention the implicit vs explicit approaches. In Section 3 at first we define the quasi-periodic granularities and propose an abstract implicit representation of quasi-periodic granularities, and then we propose an extended relational temporal data model coping with it. Temporal range queries are particularly important in the temporal Database context [17]. In Section 4 we identify different types of temporal range queries in the context of periodic data, and we devise algorithms to cope with them. In section 5, we briefly overview the current approaches to index temporal data, proposing the adoption of TD-tree for indexing our representation. In section 6, we present an experimental evaluation of our approach, showing its advantages with respect to the 'traditional' explicit approach. In section 7 we provide proof that proposed algorithm is correct and complete. Finally, section 8 addresses conclusions.

\section{Implicit vs. Explicit Approaches to Periodic Data}

There is an obvious and trivial way to cope with user-defined periodic data, namely by explicitly storing all of them. Such an approach, usually called 'explicit' (or 'extensional') approach, basically reduces periodic data to standard temporal non-periodic ones. For instance, in order to deal with an activity $X$ scheduled each day from 9 to $11 \mathrm{am}$ in a year, an explicit approach can simply represent the valid time of the activity through list all of the 365 periods in which the activity takes place. The obvious advantage of such an approach is its simplicity as periodic temporal data are simply coped with as standard temporal data, so that any temporal Database approach in the literature can suffice. Such approach makes all of the database implementation simpler, from indexing to query processing. However, there are at least three main disadvantages of the 'explicit' approach:

- It is not 'commonsense' and 'human-oriented': humans usually tend to abstract, so they usually prefer to manage periodic data in an implicit way. For instance, every Monday this semester from 9 to $11 \mathrm{am}$.

- It is not feasible in the case of 'open-ended' periodic data, when the duration of repetitions is unknown, so that no explicit elicitation of all the data is possible. 
- It can be very expensive in regard to space complexity. In many practical application areas the number of repetitions of activities is very high, so making explicit all the repetitions would be very space demanding. For example, activities on an automatized schedule in a production chain may be performed at a very high (periodic) frequency for very long duration of repetitions. Making all such data explicit might rapidly reach a critical data size even for the most efficient commercial DBMS, with dramatic consequences especially in term of physical disk I/O's.

\section{Quasi-Periodic Granularities}

We rely on the definition of granularity taken from the temporal database glossary [12] and adapted in [18], [19]. Such definitions are the basis for our treatment of periodic data.

Definition 1. A granularity $G$ quasi-periodically groups into a granularity $H$ if:

(i) G groups into $H$, and

(ii) there exists a finite set of finite intervals $E_{1}, \ldots, E_{z}$ and positive integers $n$ and $m$, where $n$ is less than the minimum of the number of granules of $H$, such that for all $i \in Z$, if $H(i)=\bigcup_{r=0}^{k} G\left(j_{r}\right)$ and $H(i+n) \neq \emptyset$, and $i+n<\min (E)$, where $E$ is the closest existing exception after $H(i)$ (if such exception exists; otherwise $E=\max (k \mid H(k) \neq \emptyset)$, then $H(i+n)=\bigcup_{r=0}^{k} G\left(j_{r}+m\right)$.

The definition of periodic and quasi-periodic granularities in terms of a bottom granularity is:

Definition 2. A periodic granularity is a granularity periodic with respect to the bottom granularity.

In Figure 1, we graphically show the basic notions underlying our representation. The 'Explicit Representation' part of the figure shows what a periodic data actually is a sequence of time periods, which repeat regularly on the time line. Usually repetitions are bounded and only a part of the whole timeline, which we term Frame Time is considered. Given some periodic activity, a range query may ask whether such an activity has to be performed within a specific time period $Q$ or not. If we have an explicit representation of all the time periods for the periodic activity, the range query can be easily answered by looking whether there is an intersection between explicitly stored data and query window $Q$.

Considering the implicit representation, one can isolate the pattern of periods that repeat regularly in time. Only one such pattern must be explicitly stored, with the intended meaning that such a pattern repeats regularly every $P$ during the Frame Time. It is obvious that the the implicit representation is space-effective, but answering the above query on the basis of the implicit representation is quite complex, since standard checks for intersections between the data and query window cannot be applied. Such a challenging task will be addressed in this work. Analogous challenging problems have to be faced when coping with the other kind of queries (e.g., queries based on algebraic relational operators) on an implicit representation of periodic data. 

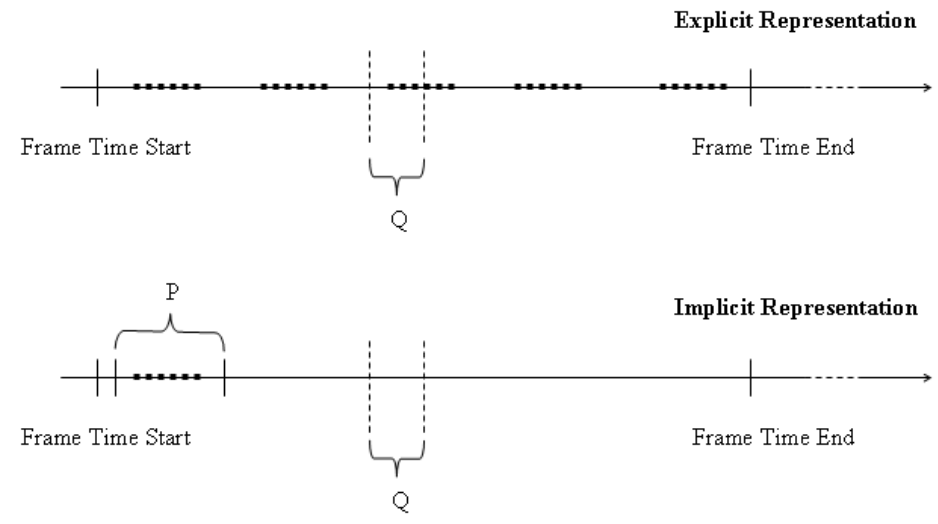

Fig. 1: Representations of Periodic data

\subsection{An implicit Representation of Quasi-Periodic Granularities}

As a working example, let us consider the following user-defined granularity. Let us suppose that, in the year 2012, starting from Monday January 9th and ending on Sunday December 23rd, the 'working shift' for one employee in company is from 08:00 to 12:00, and from 14:00 to 18:00 each day from Monday to Friday, and from 08:00 to 12:00 on Saturday, let us call such a granularity 'WS'. In addition, let us suppose that person also works on Saturday evening from 14:00 to 18:00 in two specific days, say on January $14^{\text {th }}$ and $21^{\text {st }}$, let us call 'WS+' the granularity WS with such an addition.

We have that, by definition, each quasi-periodic granularity groups quasi-periodically with respect to a bottom granularity, we will assume that hours (henceforth 'HR') is the bottom granularity (but our approach is independent of such a choice). Let us consider the user-defined granularity WS. First of all, notice that each granule in WS is composed by a set of granules of HR. For instance, the first granule WS(0) of WS is the union of the granules HR(176), HR(177), HR(178), and HR(179), which represent working shift first Monday from 8:00 to 12:00, and HR(182), HR(183), HR(184), and HR(185), which represents working shift on first Monday from 14:00 - 18:00 (assuming to denote with $\operatorname{HR}(0)$ the first hour of January $\left.1^{s t}, 2012\right)$. An instance of the 'grouping patterns' is:

$$
\begin{aligned}
\mathrm{WS}(0)=\{ & \operatorname{HR}(176) \cup \mathrm{HR}(177) \cup \operatorname{HR}(178) \cup \operatorname{HR}(179) \cup \\
& \operatorname{HR}(182) \cup \operatorname{HR}(183) \cup \operatorname{HR}(184) \cup \operatorname{HR}(185)\} \\
& \ldots \\
\mathrm{WS}(5)=\{ & \operatorname{HR}(296) \cup \operatorname{HR}(297) \cup \operatorname{HR}(298) \cup \operatorname{HR}(299)\}
\end{aligned}
$$

This pattern repeats every 168 granules of HR (each week), and 'frame time' the period of time spanning from the first and last non-empty granules in $H$ (8567 HR). However, such an initial representation can be simplified:

(a) If all granularities are expressed in terms of the bottom granularity, the bottom granularity may be left implicit. In our example, the representation of the first item 
may be simplified, stating, e.g., $\mathrm{WS}(0)=\{176,177,178,179,182,183,184,185\}$ and so on.

(b) Second, contiguous granules of the bottom granularity can be more compactly represented as (convex) periods. For instance, in our example, the representation of the first item may be simplified, stating, e.g., $\mathrm{WS}(0)=\{[176,179],[182,185]\}$ and so on.

(c) Third, given a periodic granularity any 'periodic pattern' can be chosen in order to represent it. However, if we adopt the convention that the chosen 'periodic pattern' is the first one starting at the granule ' 0 ' of the bottom granularity $(\operatorname{HR}(0)$ in our example), then also the indexes of the granules of the quasi-periodic granularity may be left implicit.

Notice that simplifications (a) and (c) together are very important, since they allow us to keep all the indexes implicit in the representation, making it much more compact and easy. Finally, in the case of quasi-periodic granularities, a further component must be considered into the representation, namely, the list of the non-periodic repetitions. In other words, besides time periods which repeats periodically in time, we also optionally add a set of periods that do not follow such periodic pattern.

We propose the following implicit representation of a quasi-periodic granularity $G$. A quasi-periodic granularity $G$ is represented by a quadruple:

$$
G=\left\langle P, I_{P}, I_{A}, F T\right\rangle
$$

where $P$ is an integer representing the duration of the periodic pattern; $I_{P}$ is the set of the convex periods in the first 'periodic pattern' of the bottom granularity; $I_{A}$ is the set of the convex periods constituting the aperiodic part; $F T$ is a period constituting the frame time. In turn, a period having as first granule the bottom granule $B(i)$ and as last granule the bottom granule $B(j)$ is represented by ' $[i, j]$ '.

Working shift example, WS and WS+ are represented in our formalism as follows:

$$
\begin{aligned}
\mathrm{WS}=\langle & 168,\{[176,179],[182,185],[200,203], \ldots,[296,299]\}, \\
& \{\},[168,8567]\rangle \\
\mathrm{WS}+= & \langle 168,\{[176,179],[182,185],[200,203], \ldots,[296,299]\}, \\
& \{[2029,2033],[2197,2201]\},[168,8567]\rangle
\end{aligned}
$$

\subsection{Data Model for Implicit Periodic Data}

The abstract representation of quasi-periodic granularities presented in previous subsection is the basis to define our extended model, coping with quasi-periodic data in a relational environment. However, several aspects need to be investigated, and choices done. For instance, we could associate an unique identifier to each user-defined quasiperiodic granularity, and extend the data model with just an additional attribute, used in order to pair each tuple with the identifier of the granularity. One (or more) dedicated tables could then be used in order to associate with each identifier the 'implicit' description of the granularity they denote. Our model is also based on the two considerations: 
- Given a periodic tuple, its 'frame time' can be interpreted, roughly speaking, as an approximation of its 'valid time', in the sense that it contains all the time periods in which the tuple holds.

- Given a quasi-periodic tuple, the 'non-periodic' part of its granularity can be simply represented by a set of time periods, i.e., of 'standard' valid times in the 'consensus' approach.

We can now define our new data model. Given any schema $R=\left(A_{1}, \ldots, A_{n}\right)$ (where $A_{1}, \ldots, A_{n}$ are standard non-temporal attributes), a periodic relation $r$ (in our example we termed Activity) is a relation defined over the schema

$$
R^{P}=\left(A_{1}, \ldots, A_{n} \mid V T_{S}, V T_{E}, \text { Per, PatID }\right)
$$

where:

- $V T_{S}$ is a timestamp representing the starting point of the 'frame time'

- $V T_{E}$ is a timestamp representing the ending point of the 'frame time'

- Per is an interval, representing the duration of the repetition pattern

- PatID is an identifier, denoting a periodic pattern

In addition, in order to code periodic patterns, an additional dedicated relation (a valid-time relation, in the sense of TSQL2) is needed (called Pattern relation). In Table 1 'NULL' in column Per indicates that the tuples represent aperiodic component as there is no periodic repetitions and also it does not refer to periodic pattern.

The Pattern is a relation over the schema (PatID, Start, End), where PatID is an attribute containing identifiers denoting periodic patterns, while Start and End are temporal attributes denoting the starting and the ending points of the periods in the periodic pattern.

\begin{tabular}{|l|l|l|l|l|l|l|}
\hline ActID & Act & ActorID & $V_{S}$ & $V_{\text {E }}$ & Per & PatID \\
\hline 1 & A & John & 168 & 8567 & 168 & P1 \\
\hline 2 & A & John & 2029 & 2033 & Null & Null \\
\hline 3 & A & John & 2197 & 2201 & Null & Null \\
\hline
\end{tabular}

Table 1: Activity periodic relation - Implicit model

\begin{tabular}{|l|l|l|}
\hline PatID & Start & End \\
\hline$P 1$ & 176 & 179 \\
$\cdots$ & $\cdots$ & $\cdots$ \\
$P 1$ & 296 & 299 \\
\hline
\end{tabular}

Table 2: Pattern relation - Implicit model 


\section{Range Queries about Implicit Periodic Data}

In the context of periodic data represented in implicit way, we identified three different types or range queries, depending on whether:

- One is interested in the non-temporal part of the tuples only. For example what activities have to be performed from May 1 st to July 31, 2012, and by which employees? To this type of queries we will refer as 'atemporal range queries' henceforth.

- One is interested in the tuples and in their explicit time. Such as what activities have to be performed from May 1st to July 31, 2012, by which employees; for each of them, list all the periods they have to be performed - within the query period; We will refer as 'explicit temporal range queries'.

- One is interested in the tuples and in their implicit time. What activities have to be performed from May 1st to July 31, 2012, by which employees, and when -implicit time? This is 'implicit temporal range queries'.

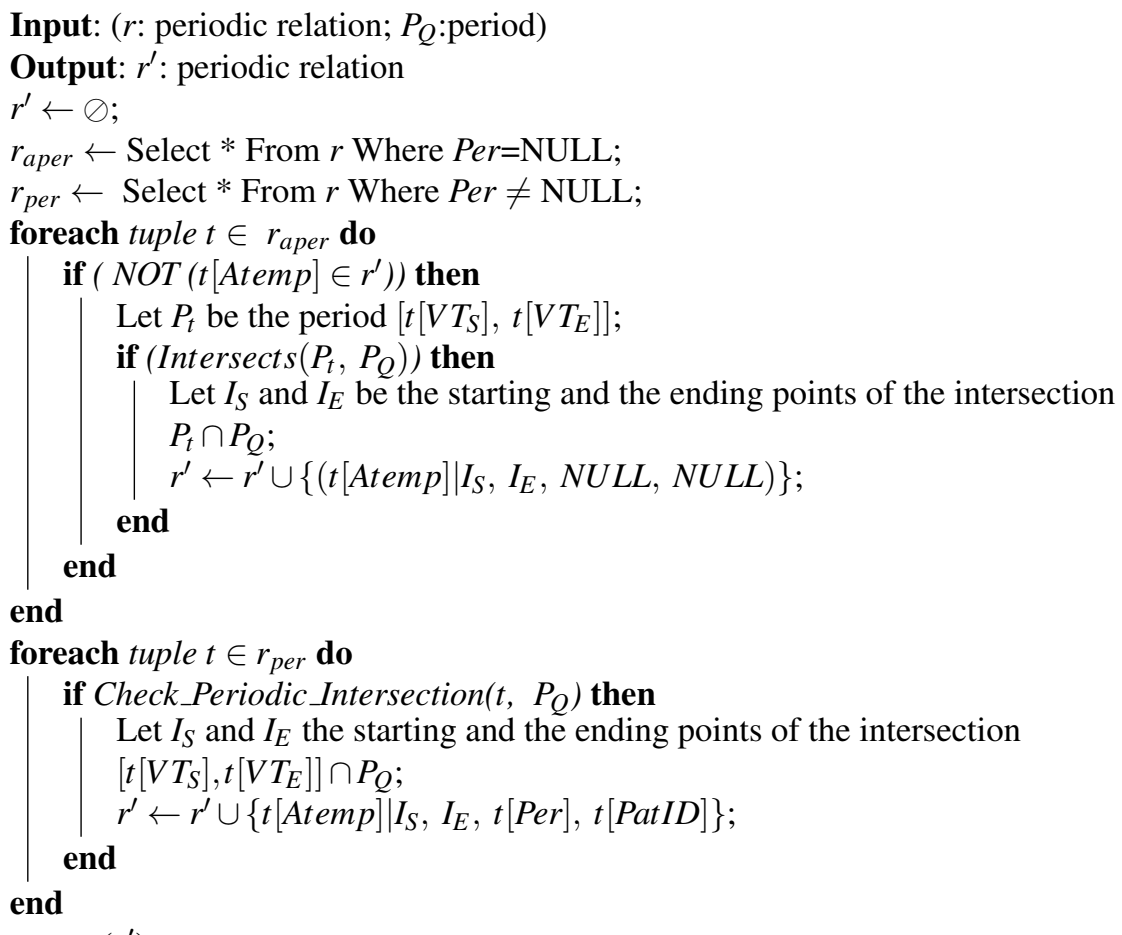

Algorithm 1: Implicit temporal range queries

Algorithms for 'atemporal range queries' can be found in [18] and 'explicit temporal range queries' on implicit model is straight forward by making explicit answer from 'implicit temporal range queries'. In following subsection we provide algorithm for implicit temporal range queries. 


\subsection{Implicit Temporal Range Queries}

We describe at an abstract level the algorithm for implicit temporal range queries. The Algorithm 1 takes as input a implicit periodic relation $r$ and a query period $P_{Q}$ and gives as output a periodic relation containing all the tuples occurring during $P_{Q}$, answer is provided in implicit representation. Only parts of the valid times that intersect with the query period $P_{Q}$ are reported in output. To perform implicit temporal range queries there is need to check for intersection and Algorithm 2 Check_Periodic_Intersection has as input a periodic tuple $(\operatorname{PerID} \neq N U L L)$, and the query period $P_{Q}$, and checks whether there is an intersection.

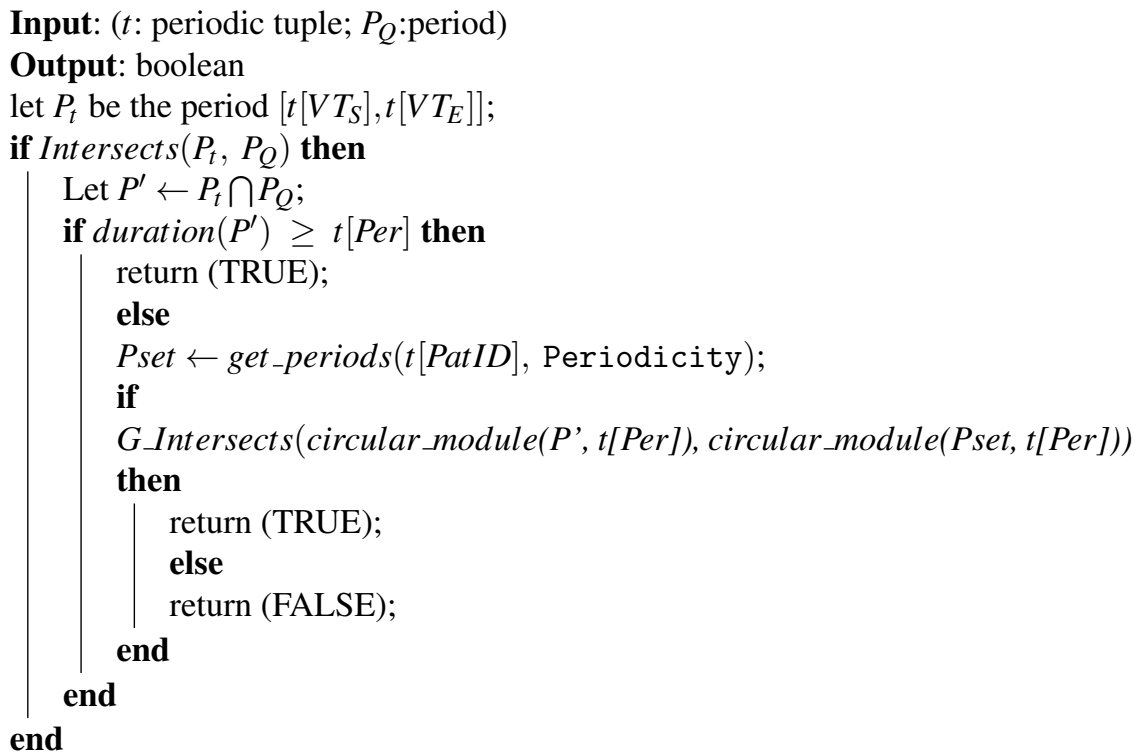

Algorithm 2: Check Periodic Intersection

There is also required to perform circular module, provided in Algorithm 3, to identify which patterns might intersect with query interval.

Input: Set : set_of_periods, $n$ : int

Output: res: set_of_periods

res $\leftarrow\{\}$;

foreach $[s, e] \in$ Set do

$s^{\prime} \leftarrow s \bmod n ;$

$e^{\prime} \leftarrow e \bmod n$

if $\left(s^{\prime} \leq e^{\prime}\right)$ then

res $\leftarrow \operatorname{res} \cup\left\{\left[s^{\prime}, e^{\prime}\right]\right\} ;$

else

res $\leftarrow \operatorname{res} \cup\left\{\left[0, e^{\prime}\right],\left[s^{\prime}, n-1\right]\right\} ;$

end

end

return (res)

Algorithm 3: Circular module 
If $n=7$ and Set_of_periods $=\{[11,13]\}$ then circular_module $($ Set_of_periods, $n)=$ $\{[4,6]\}$, while if Set_of_period $=[11,16]$ we have that $s^{\prime}=11 \bmod 7=4$ and $e^{\prime}=$

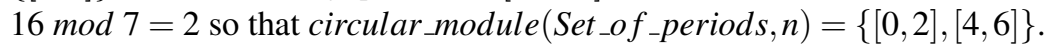

\section{Indexing Periodic Data}

Different access methods have been presented in literature and some of them have been recommended for handling temporal data. Because we intend to index temporal data within commercial relational DBMS, we can only considered ones which can exploit existing structures such as the $\mathrm{B}^{+}$tree and do not require any modification to the database kernel. Several such structures have been mentioned in literature. Such as methods which can map one dimensional ranges to one dimensional points, MAP21 [20], or manage the intervals by two relational indexes RI-tree method [21]), or partition the space and utilize the virtual structure TD-tree [22], [23].

The Triangular Decomposition Tree (TD-tree) is efficient access method for temporal data. In contrast to previously proposed access methods for temporal data this method can efficiently answer a wide range of query types, including point queries, intersection queries, and all nontrivial interval relationships queries, using a single algorithm without dedicated query transformations. It also can be built within commercial relational database system as it uses only builtin functionalities within the SQL:1999 standard and therefore no modification to the database kernel is required. The TD-tree is a space partitioning access method. The basic idea is to manage the temporal intervals by a virtual index structure [22].

Since we intend to index temporal periodic data (both implicit and explicit) and periodic data are temporal in nature, we will utilize methods for indexing temporal data. It has been shown in the literature that the TD-tree [22] has the best performance considering the Physical disk I/O and the query response time and at the same time can be employed within commercial RDBMS, we decided to employ the TD-tree in our implementation. Specifically, we index the $V T_{S}$ and $V T_{E}$ temporal attributes of periodic relations.

However it is worth stressing that any method, previously proposed in literature for indexing intervals, can deal only with explicit times. As a consequence, they can not be applied directly to any 'implicit' approach to periodic data, since in such approaches periods are only implicitly described. As a matter of facts, one of the main challenges of our approach was that of providing a suitable querying approach, which in conjunction with indexing methodology for indexing intervals can efficiently manage implicit periodic data.

\section{Empirical testing}

In order to show the practical relevance of our implicit approach to efficiently manage periodic data, we have performed an extensive experimental evaluation. In particular, we have compared the performance of our approach with respect to the standard explicit one. We used Oracle built-in methods for statistics collection, analytic SQL functions, and the PL/SQL procedural runtime environment. We compared our results considering 
the space usage, physical I/O, CPU usage, and query response time. In order to carry on the experiments, the same periodic activities concerning sample hospital patients have been represented both in the implicit and explicit model and we performed atemporal range queries as well as detail experiments to investigate the explicit/implicit ratio when implicit approach starts to perform better. The experiments have shown that already at explicit/implicit ratio of 5 our implicit method starts to gain significant advantages as regards to both disk I/O and query response time. It is important to mention that the ratio in example where we simulated small hospital was 157 . More details of other experiments can be found in [18].

\section{Correctness and Completeness of the method}

In order to prove that the range query answering algorithms, operating on the extended temporal model, are correct and complete, we show that, given any range query and a database of relations expressed in our implicit data model, our algorithms provide all and only the results that are provided by asking the same queries on an explicit representation of the same data.

In the proof we will use the notation above, plus the following conventions: $Q_{\operatorname{Imp}}$ is the set of tuples returned by algorithm 1 given an atemporal range query $Q$; similarly $Q_{E x p}$ is the set of tuples from Exp that satisfy the temporal range query $Q$. To avoid confusion with interval we will use the dot notation for temporal attributes of tuples apart for the non temporal part, for which we continue to use $t[$ Atemp $]$. In addition to improve readability we will use $c \_$module instead of circular_module.

Let us at first define the relationships between the implicit and the explicit models. Let Imp be the join of a relation $r$ (Activity) and the table Pattern in the implicit model, and Exp be the corresponding relation in the explicit model. Then, given Imp, Exp is defined as follows:

$$
\begin{aligned}
\operatorname{Exp}=\{( & \left.\left(t[\text { Atemp }], t . V T_{s}, t . V T_{e}\right): t \in r \wedge t . p e r=N U L L\right\} \cup \\
& \left\{\left(t[\text { Atemp }], i^{-}, i^{+}\right): \exists n \in \mathbb{N},\right. \\
& \left.t . V T_{s} \leq n * t . p e r+(t . s t a r t-t . o f f) \leq t . V T_{e}\right\}
\end{aligned}
$$

where $\forall n \in \mathbb{N}:\left\lfloor t . V T_{s} / t\right.$.per $\rfloor \leq n \leq\left\lfloor t . V T_{e} / t\right.$.per $\rfloor$

$$
\begin{aligned}
& i^{-}=\max \left\{t . V T_{s}, n * t . p e r+(t . s t a r t-t . o f f)\right\} \\
& i^{+}=\min \left\{t . V T_{e}, n * t . p e r+(t . e n d-t . o f f)\right\}
\end{aligned}
$$

and $t$.off $=\lfloor$ t.start $/$ t.per $\rfloor * t$.per.

Soundness. To prove that algorithm 1 is sound we have to prove that if $t \in Q_{I m p}$, then $\exists s \in \operatorname{Exp}$, such that $t[$ Atemp $]=s[$ Atemp $]$ and $s \in Q_{\text {Exp }}$. Given algorithm 1, we have to consider two mutually exclusive cases: (1) $t \in r_{\text {aper }}$ and (2) $t \in r_{\text {per }}$.

Case 1: t.per $=N U L L$, and by construction the tuple $s=\left(t[\right.$ Atemp $\left.], t . V T_{s}, t . V T_{e}\right)$ is in Exp, and $\left[t . V T_{s}, t . V T_{e}\right] \cap P_{Q} \neq \emptyset$, thus $s \in Q_{E x p}$ such that $t[$ Atemp $]=s[$ Atemp $]$.

Case 2: We have two mutually exclusive subcases: (i) duration $\left(P^{\prime}\right)>t$.per, and (ii) duration $\left(P^{\prime}\right) \leq$ t.per. 
For (i): Let $n=\left\lfloor P^{-} / t\right.$.per $\rfloor, n+m=\left\lfloor P^{\prime+} / t . p e r\right\rfloor, m \geq 1$ and $o=\left\lfloor t . V T_{s} / t . p e r\right\rfloor$. Let pat $_{j}=\left(\right.$ start $_{j}$, end $\left._{j}\right)$ be any pattern in the table Pattern. Let

$$
\begin{aligned}
& j^{-}=(n-o) * t . p e r+\text { start }_{j}-t . o f f \\
& j^{+}=\min \left\{(n-o) * t . p e r+\left(\text { end }_{j}-t . o f f\right), t . V T_{e}\right\} .
\end{aligned}
$$

If $j^{-} \geq P^{\prime-}$, then we consider the tuple $s=\left(t[\right.$ Atemp $\left.], j^{-}, j^{+}\right)$. Now $s \in \operatorname{Exp}$ since $t . V T_{s} \leq P^{\prime-} \leq j^{-}$and $\left[j^{-}, j^{+}\right]<t . p e r$, so $t . V T_{e} \geq P^{\prime+} \geq j^{+}$, and for essentially the same reasons, $s \in Q_{E x p}$. If $j^{-}<{P^{\prime}}^{-}$, then we can repeat the same argument as the previous case, but this time we set

$$
\begin{aligned}
& j^{-}=(n+1-o) * t . p e r+\left(\text { start }_{j}-t . o f f\right), \\
& j^{+}=\min \left\{(n+1-o) * t . p e r+\left(\text { end }_{j}-t . o f f\right), t . V T_{e}\right\} .
\end{aligned}
$$

For (ii): Let $p a t_{j}=\left[\right.$ start $_{j}$, end $\left._{j}\right]$ be a pattern in the table Pattern such that $c \_$module $\left(P^{\prime}, t . p e r\right)$ $\cap$ c.module $\left(\right.$ pat $\left._{j}, t . p e r\right) \neq \emptyset$. Let $n^{ \pm}=\left\lfloor P^{ \pm \pm} / t\right.$.per $\rfloor, r^{-}=\left\lfloor\right.$start $t_{j} / t$.per $\rfloor$ and $r^{+}=$ $\left\lfloor\right.$ end $_{j} /$ t.per $\rfloor$. Then

$$
\begin{aligned}
& \text { c_module }\left(P^{\prime}, \text { t.per }\right)= \begin{cases}{\left[q^{-}, q^{+}\right]} & \text {if } n^{+}=n^{-} \\
{\left[0, q^{+}\right],\left[q^{-}, t . p e r-1\right]} & \text { otherwise }\end{cases} \\
& \text { c.module }\left(\text { pat }_{j}, t . p e r\right)= \begin{cases}{\left[o^{-}, o^{+}\right]} & \text {if } r^{+}=r^{-} \\
{\left[0, o^{+}\right],\left[o^{-}, t . p e r-1\right]} & \text { otherwise }\end{cases}
\end{aligned}
$$

Since the intersection of $c_{\_}$module $\left(P^{\prime}\right.$, t.per $)$ and $c \_$module $\left(\right.$pat ${ }_{j}$, t.per $)$ is not empty, we have the following cases (where $E=t$.per -1 ):

1. $\left[q^{-}, q^{+}\right] \cap\left[o^{-}, o^{+}\right] \neq \emptyset$ or

2. $\left[q^{-}, q^{+}\right] \cap\left[0, o^{+}\right] \neq \emptyset$ or

3. $\left[q^{-}, q^{+}\right] \cap\left[o^{-}, E\right] \neq \emptyset$ or

4. $\left[0, q^{+}\right] \cap\left[o^{-}, o^{+}\right] \neq \emptyset$ or

5. $\left[0, q^{+}\right] \cap\left[0, o^{+}\right] \neq \emptyset$ or

6. $\left[0, q^{+}\right] \cap\left[o^{-}, E\right] \neq \emptyset$ or

7. $\left[q^{-}, E\right] \cap\left[o^{-}, o^{+}\right] \neq \emptyset$ or

8. $\left[q^{-}, E\right] \cap\left[0, o^{+}\right] \neq \emptyset$ or

9. $\left[q^{-}, E\right] \cap\left[o^{-}, E\right] \neq \emptyset$.

Let us consider the tuple $e=\left(t[\right.$ Aper $\left.], j^{-}, j^{+}\right)$where, $n$ and $r$ can be set to either $n^{+}$or $n^{-}$and $r^{+}$or $r^{-}$depending on the cases above:

$$
\begin{aligned}
& j^{-}=\max \left\{(n-r) * t . p e r+t . \text { start }_{j}-t . \text { off }, t . V T_{s}\right\} \\
& j^{+}=\min \left\{(n-r) * t . p e r+t . \text { end }_{j}-t . \text { off }, t . V T_{e}\right\}
\end{aligned}
$$

It is immediate to verify that $e \in \operatorname{Exp}$ (since the tuple in Imp satisfies the query $n-r \geq 0$ for the appropriate assignment of $n$ and $r$ ). Cases 2-9 are similar to the case above. What we have to do is to consider the appropriate values of $n$, where we have to consider $n$ or $n+1$ depending whether the circular module returns one or two intervals. 
Completeness. To prove completeness we have to prove that if $t \in Q_{E x p}$, then $\exists s \in$ Imp , such that $t[$ Atemp $]=s[$ Atemp $]$ and $s \in Q_{\text {Imp }}$. We prove the property by contradiction. Suppose that the property does not hold. This means that for every tuple in $t \in Q_{E x p}$ there is not tuple in Imp such that the tuple in Imp generated the tuple $t$, but the tuple in Imp does not satisfy the query.

Clearly the tuple $s$ in Imp cannot be an aperiodic tuple, otherwise we obtain immediately a contradiction, since the $s . V T_{s}=t . V T_{s}$ and $s . V T_{e}=t . V T_{e}$, and the conditions for the explicit query and the condition in the algorithm for the implicit query are the same. Similarly we have that s.per $\left\langle P^{\prime}\right.$; otherwise $s$ satisfies the condition to be included in $Q_{\text {Imp }}$, contrary to the assumption.

\section{Conclusions}

In this work, we presented a new approach to cope efficiently with periodic data in relational databases. Specifically:

- we have presented an 'implicit' relational data model for user-defined periodic data, which is based on the 'consensus' definition of granularity in the temporal database glossary and its extension to cover periodic granularities, and it is a consistent extension of TSQL2's.

- we have taken into account range queries, providing sound and complete query answering algorithms for them;

- we have extended Codd's algebraic operators of Cartesian product, Union, Projection, nontemporal selection, and Difference, in order to provide a complete query language coping with implicit periodic data; Such operators are sound and complete, and are a consistent extension of BCDM (and TSQL2) algebra. Details can be found [18].

- in an extensive experimentation of our model and methodology, we showed that our 'implicit' approach overcomes the performance of traditional 'explicit' approaches both in terms of space and disk I/O's, and in terms of query response time. Moreover, we have also analysed to what extent our implicit approach is advantageous, depending on the 'explicit/implicit' ratio.

\section{Acknowledgements}

The author is very grateful to Richard T. Snodgrass for many in-dept and inspiring comments on a preliminary version of this work. Also, author would like to acknowledge Paolo Terenziani and Guido Governatori for contributions on algorithms and proofs.

\section{References}

1. Soo, M., Snodgrass, R.: Multiple calendar support for conventional database management systems. In: Proc. Int'1 Workshop on an Infrastructure for Temporal Databases. (1993)

2. Terenziani, P.: Temporal periodicity. In: Encyclopedia of Database Systems, Springer Verlag, Ling Liu and M. Tamer zsu Editors (2009) 
3. Chomicki, J., Imielinski, T.: Finite representation of infinite query answers. ACM ToDS 18(2) (1993) 181-223

4. Kabanza, F., Stevenne, J.M., Wolper, P.: Handling infinite temporal data. Journal of Computer and System Sciences 51 (1995) 3-17

5. Leban, B., McDonald, D., Forster, D.: A representation for collections of temporal intervals. In: Procs. of AAAI'86. (1986) 367-371

6. Niezette, M., Stevenne, J.M.: An efficient symbolic representation of periodic time. In: Procs. of CIKM. (1992)

7. Bettini, C., De Sibi, R.: Symbolic representation of user-defined time granularities. Annals of Mathematics and Artificial Intelligence 30(1-4) (2000) 53-92

8. Ning, P., Wang, X., Jajodia, S.: An algebraic representation of calendars. Annals of Mathematics and Artificial Intelligence 36(1-2) (2002) 5-38

9. Terenziani, P.: Symbolic user-defined periodicity in temporal relational databases. IEEE TKDE 15(2) (2003) 489-509

10. Egidi, L., Terenziani, P.: A flexible approach to user-defined symbolic granularities in temporal databases. In: Procs. of ACM SAC'05. (2005) 592-597

11. Egidi, L., Terenziani, P.: A mathematical framework for the semantics of symbolic languages representing periodic time. Annals of Mathematics and Artificial Intelligence 46(3) (2006) 317-347

12. Bettini, C., Dyreson, C., Evans, W., Snodgrass, R., Wang, X.: A glossary of time granularity concepts. In O. Etzion, S. Jajodia, and S. Sripada, editors, Temporal Databases: Research and Practice, number 1399 in LNCS State-of-the-art Survey, Springer-Verlag (1998) 406-413

13. Snodgrass, R.T., Ahn, I.: A Taxonomy of Time in Databases. In Navathe, S.B., ed.: Proceedings of the 1985 ACM SIGMOD International Conference on Management of Data, ACM Press (1985) 236-246

14. Snodgrass, R.T.: The TSQL2 Temporal Query Language. Kluwer Academic (1995)

15. Dyreson, C.E., Snodgrass, R.T., Freiman, M.: Efficiently Supporting Temporal Granularities in a DBMS. Technical Report TR 95/07 (1995)

16. Bettini, C., Jajodia, S., Wang, S.: Time Granularities in Databases, Data Mining, and Temporal Reasoning. Springer Verlag (2000)

17. Tsotras, V.J., Jensen, C.S., Snodgrass, R.T.: An Extensible Notation for Spatiotemporal Index Queries. ACM SIGMOD Record 27(1) (1998) 47-53

18. Terenziani, P., Stantic, B., Bottrighi, A., Sattar, A.: An intensional approach for periodic data in relational databases. Journal of Intelligent Information Systems - Online first: DOI 10.1007/s10844-013-0245-8 (2013)

19. Stantic, B., Terenziani, P., Governatori, G., Bottrighi, A., Sattar, A.: An implicit approach to deal with periodically-repeated medical data. Journal in Artificial Intelligence in Medicine 55(3) (2012) 149-162

20. Nascimento, M.A., Dunham, M.H.: Indexing Valid Time Databases via $B^{+}$-Tree. IEEE Transactions on Knowledge and Data Engineering 11(6) (1999) 929-947

21. Kriegel, H.P., Ptke, M., Seidl, T.: Managing intervals efficiently in object-relational databases. Proceedings of the 26th International Conference on Very Large Databases (2000) 407-418

22. Stantic, B., Terry, J., Topor, R., Sattar, A.: Advanced indexing technique for temporal data. In: Computer Science and Information Systems - ComSIS. Volume 7. (2010) 679-703

23. Stantic, B., Terry, J., Topor, R., Sattar, A.: Indexing Temporal Data with Virtual Structure. In: in Advances in Databases and Information Systems - ADBIS2010. (2010) 591-594 